\title{
Evidence for a generic interceptive strategy
}

\author{
DENNIS M. SHAFFeR \\ Ohio State University, Mansfield, Ohio \\ Michael K. MCBeath \\ Arizona State University, Tempe, Arizona \\ SCOTT M. KRaUchunas \\ Saint Anselm College, Manchester, New Hampshire \\ AND \\ ThOMas G. SugaR \\ Arizona State University, Tempe, Arizona
}

\begin{abstract}
In the present work, we first clarify a more precise definition of instantaneous optical angles in control tasks such as interception. We then test how well two interceptive strategies that have been proposed for catching fly balls account for human Frisbee-catching behavior. The first strategy is to maintain the ball's image along a linear optical trajectory (LOT). The second is to keep vertical optical ball velocity decreasing while maintaining constant lateral optical velocity. We found that an LOT accounted for an average of over $96 \%$ of the variance in optical Frisbee movement, while maintenance of vertical and lateral optical velocities was random. This work confirms a common interception strategy used across interceptive tasks, extending to complex target trajectories.
\end{abstract}

With relatively little practice, millions of recreational players become adept at intercepting targets such as baseballs, footballs, and Frisbees, even when the objects travel through trajectories that dramatically change directions in midflight. In the present study, we test whether humans catching Frisbees utilize the same simple viewer-based navigational heuristics that have been established for baseball and cricket fielders catching fly balls (McBeath, Shaffer, \& Kaiser, 1995, 1996; McLeod, Reed, \& Dienes, 2001, 2003, 2006). When baseball outfielders run to catch fly balls, they use natural, geometrically invariant properties to optically maintain control over the ball. When balls are headed off to the side, fielders select a running path that maintains a linear optical trajectory (LOT) for the ball relative to home plate and the background scenery that guides them to catch the ball. In our previous work, we found evidence supporting the premise that the optical information available to the outfielder can be simply analyzed by examining it as a unified 2-D optical image, the geometry of which is shown in Figure 1, where $\alpha$ and $\beta$ specify the vertical and lateral optical angles, respectively, between the ball and its initial optical location (home plate). $\psi$ specifies the optical trajectory projection angle, or the observed angle of ball movement in the picture plane relative to the background horizon. In short, an LOT results when the fielder's running speed and direction maintain a rate of change in the horizontal optical angle, $\beta$, that matches the rate of change in the vertical optical angle, $\alpha$. Typically, this results in the outfielder fixating the image of the ball while running along a path so as to actively rotate his vantage at a constant rate. This typically results in the fielder running fastest laterally at the start, getting a little ahead of the ball, and easing up somewhat at the end.

Previously, we have also shown that fielders catching fly balls (McBeath et al., 1995) and dogs catching Frisbees (Shaffer, Krauchunas, Eddy, \& McBeath, 2004) simultaneously maintain a constant increase in the tangent of the vertical optical angle, $\tan \alpha$, which serves as a complementary cue to optical linearity. This is also the strategy used when balls are launched in the plane directly toward the fielder (McLeod et al., 2001). Although the LOT does not require a constant increase in $\tan \alpha$, it does have a looser temporal requirement that keeps it monotonically increasing (Dannemiller, Babler, \& Babler, 1996). Such navigational strategies as the LOT have been proposed as generic strategies geometrically constrained to ensure collision between a pursuer and its target, including collision of airplanes and boaters tracking another craft, dragonflies and bats chasing prey, and dogs catching Frisbees (Jablonski, 1999; McBeath et al., 1995; Olberg, Worthington, \& Venator, 2000; Pollack et al., 1995; Shaffer et al., 2004). Gibson (1979) referred to a subset of these generic strategies as invariants - that is, information in the optic array that remains stable despite other changes in context. These serve as reliable sources of information in navi-

D. M. Shaffer, shaffer.247@osu.edu 


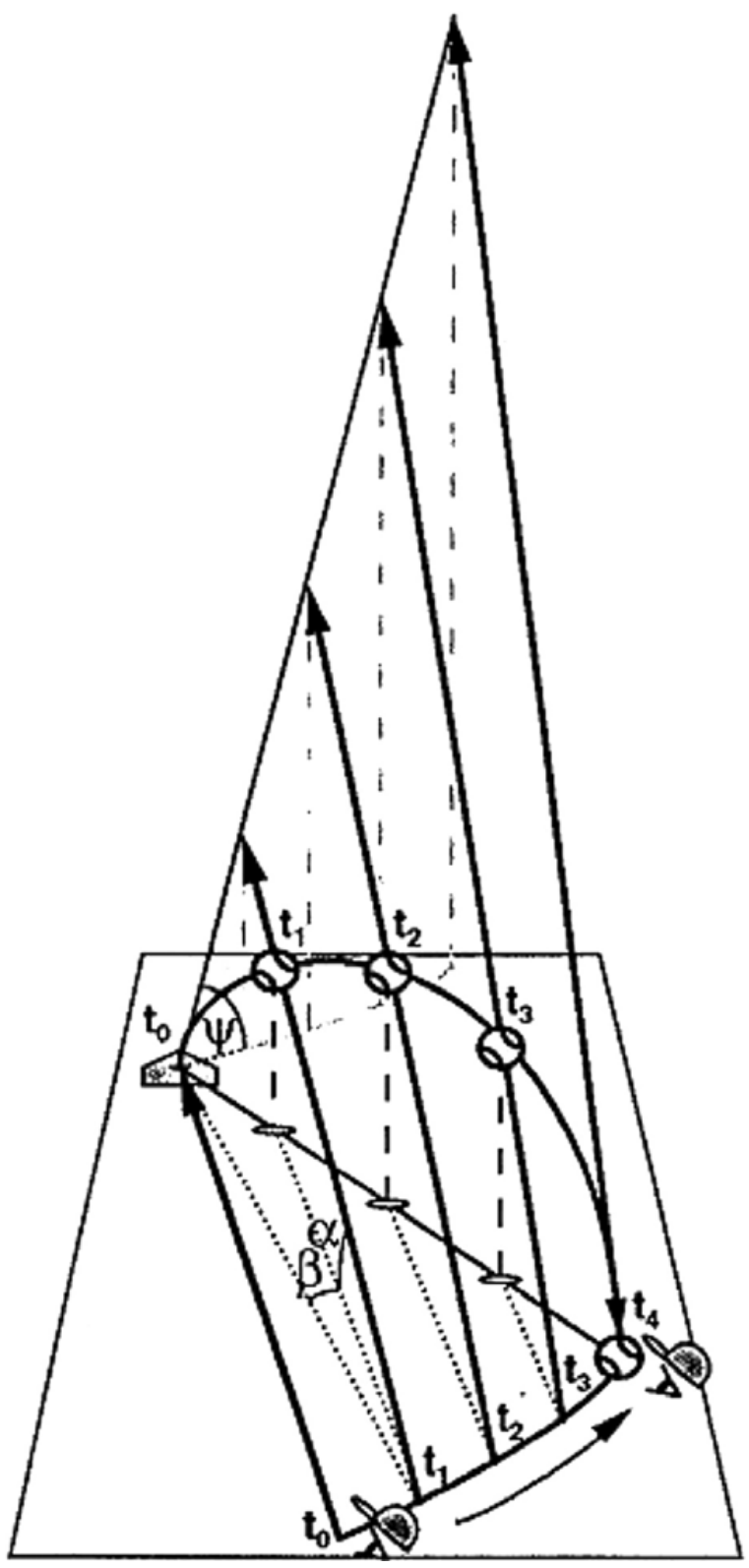

Figure 1. The linear optical trajectory (LOT) model. The LOT heuristic of maintaining a constant projection angle. The fielder selects a running path such that the lateral optical ball movement remains proportional to the vertical optical ball movement. Because equal lateral optical angles span smaller distances for nearer objects, the fielder ends up slowing down laterally as the ball approaches. The resultant running path curves slightly and circles under the ball.

gating through the world. Thus, testing the viewer-based navigational strategies such as the LOT across changes in domains (e.g., baseball vs. Frisbee catching) is an important test of the generality and reliability of the heuristic. It seems more likely that humans adopted an interception strategy for baseball that they evolved to take advantage of across a number of domains, and less likely that fielders have adopted a "baseball specific" (or "cricket specific") strategy. In this article, we examine whether a monotonically increasing LOT and a more recent proposal for catching fly balls (McLeod et al., 2006) are generic tracking strategies used by humans catching Frisbees in addition to catching fly balls. Before testing how well these two strategies can explain Frisbee-catching behavior, we first clarify the angular notation we use to describe patterns of instantaneous optical position and velocity for the LOT. The following section describes optical angles and angular and temporal constancy of planar versus cylindrical and spherical projection geometry. We argue in support of a spherical interpretation of the optical angles, coupled with the angles being defined local to the ball rather than definitions emphasizing tangents of angles relative to the ball's starting location.

\section{Projection Planes and the Definition of the Vertical and Lateral Optical Angles}

Many tasks studied by visual scientists subtend visual angles in the single digits (in degrees), and these can be simply modeled using projection plane geometry. In such cases, the vertical visual angle can be simply represented as the vertical distance across the projection plane, and the lateral visual angle as the horizontal distance. For example, pixels on a computer screen viewed at a fixed distance can be linearly transformed into degrees visual angle, and approximations such as $\alpha \approx \tan \alpha$ can be used (i.e., the law of small angles), even with visual angles roughly as large as $30^{\circ}$. In keeping with this standard, researchers of baseball perception have favored using projection plane models. Yet activities like intercepting fly balls have several aspects that appear to make such projection plane geometry a poor approach, particularly for fly balls reaching vertical angles approaching $50^{\circ}-60^{\circ}$.

First, at angles approaching $60^{\circ}-90^{\circ}$, a constant rate of change in $\tan \alpha$ produces a change in $\alpha$ that slows down to nearly $1 / 10$ of the initial optical velocity (Schneider, Ehrlich, Stein, Flaum, \& Mangel, 1978; Shaffer \& McBeath, 2002); that is, although the ball is traveling the same distance, high in the air rather than close to the ground, it seems to be traveling shorter distances (i.e., although tan $\alpha$ is constant in both cases, $\alpha$ is changing).

Second, recent eye-tracking research by Oudejans, Michaels, Bakker, and Davids (1999) and by us (McBeath, Sugar, et al., 2002) provides evidence that while fielders run to intercept fly balls, they continuously turn their heads and move their eyes and keep their fovea fixated on the ball. ${ }^{1}$ Thus, a spherical projection surface would represent a better model for maintaining an orthogonal surface to the direction of viewing. Work done with robotic simulation also confirms that an active control mechanism utilizing a spherical projection plane is a more robust strategy than a passive mechanism with a planar projection is (Sugar \& McBeath, 2001; Suluh, Sugar, \& McBeath, 2001). In other words, when fielders navigate to interception, they move along a path that effectively keeps the eye angle (measured from straight ahead) toward the ball at successive moments moving at a constant rate of change; they do not keep the eye (and effective projection 
plane) fixed and try to maintain a constant rate of change of the ball image along the retina. This finding is also consistent with neurophysiological and behavioral research confirming that visually sensitive neurons in the parietal cortex encode the location of an object relative to an eyecentered reference frame (Baud-Bovy \& Viviani, 1998; Colby \& Duhamel, 1996; Colby, Duhamel, \& Goldberg, 1995; Vetter, Goodbody, \& Wolpert, 1999).

Third, the task of catching a ball uses viewer-based strategies in which the instantaneous optical movement of the ball is a function of the position of the viewer's gaze and orientation in space relative to the ball. Most researchers of baseball perception, ourselves included, have used illustrations of fielders and balls depicting a side or bird'seye view that exemplifies a world-based coordinate system with an implied projection plane (as shown in Figure 1). In the present work, we would like to specify nomenclature that best clarifies how the task of interception is performed from the viewer-based perspective of the fielder; so, instead of describing optical position of the ball relative to its origin, we more precisely define the optical trajectory as the ongoing sum of the instantaneous changes in position of the ball image relative to stationary background scenery. Thus, in all our previous work and in the present work, we code momentary directions of motion by the relative displacements of the ball's image relative to the images of other static environmental objects. Each subsequent position of the ball's image is coded relative to its previous position against environmental markings less than $1^{\circ}$ apart. The optical trajectory of the ball, then, reflects the momentary positions where the fielder is looking while running to catch the ball, relative to these background markings.

If optical position is coded by means of using a worldbased coordinate system, there is potential ambiguity in defining optical angles. For example, a ball directly off to the side and halfway up from the horizon could be described as having a position with a lateral optical angle of $90^{\circ}$ plus a vertical optical angle of $45^{\circ}$. Similarly, without a change in reference frame, the same ball directly off to the side and halfway up the horizon could be described as having a vertical optical angle of $90^{\circ}$ plus a lateral optical angle of $45^{\circ}$. This is the classic problem of ambiguity of Euler angles. This ambiguity disappears when the optical angle is defined as the sum of instantaneous changes in optical position. In the above example, the vertical and lateral optical angles would be the sum of all of the changes that the ball went through to arrive at that final position. When a head-mounted camera is used to record optical ball position, it records each instantaneous change in position between video frames against stationary background markers, and the data can be plotted as the ongoing sum of these changes. Thus, in all of our plots, the vertical and lateral optical angles $\alpha$ and $\beta$ represent the sum of all of the instantaneous changes in vertical and lateral ball position relative to stationary background markers, respectively, on the video image from the vantage of the moving fielder.

In Figure 2, we specify $\alpha_{i}$ and $\beta_{i}$ to indicate the instantaneous change in vertical and lateral ball angles. We specify $\alpha$ and $\beta$ to indicate the sum of all of these instantaneous changes relative, respectively, to a starting hori- zontal plane on the ground and a starting vertical plane crossing through the initial optical position of the origin. One could also describe vertical and optical angles of the ball by choosing angles within these starting reference planes, and these angles are specified in the figure as $\alpha_{p}$ and $\beta_{p}$. When $\beta$ and $\beta_{p}$ are described in terms of lateral pixels across a flat projection plane (shown in the back of the figure, behind the spherical and cylindrical projection planes), they subtend the same distance. In our initial algebraic equations of the LOT we used $\beta_{p}$ instead of $\beta$ because it simplified the projection plane geometry. Yet, one can see more clearly in the spherical projection, that $\beta$ is both smaller than $\beta_{p}$, and continues to diminish toward $0^{\circ}$ as $\beta$ approaches $90^{\circ}$. This clarification and promotion of the use of the viewer-based lateral angle, $\beta$ rather than $\beta_{p}$, should resolve some difficulties that others (e.g., McLeod et al., 2001) have had with the LOT heuristic. This helps unwed maintenance of lateral optical ball velocity and monitoring of the position of its origin. It also provides a geometric mechanism for how $\beta$ will shrink relative to $\beta_{p}$, as $\alpha$ increases. Finally, defining $\alpha$ and $\beta$ as the ongoing sum of all instantaneous changes in vertical and lateral optical ball position provides an unambiguous definition of optical location that better emphasizes the viewer-based nature of the interception task.

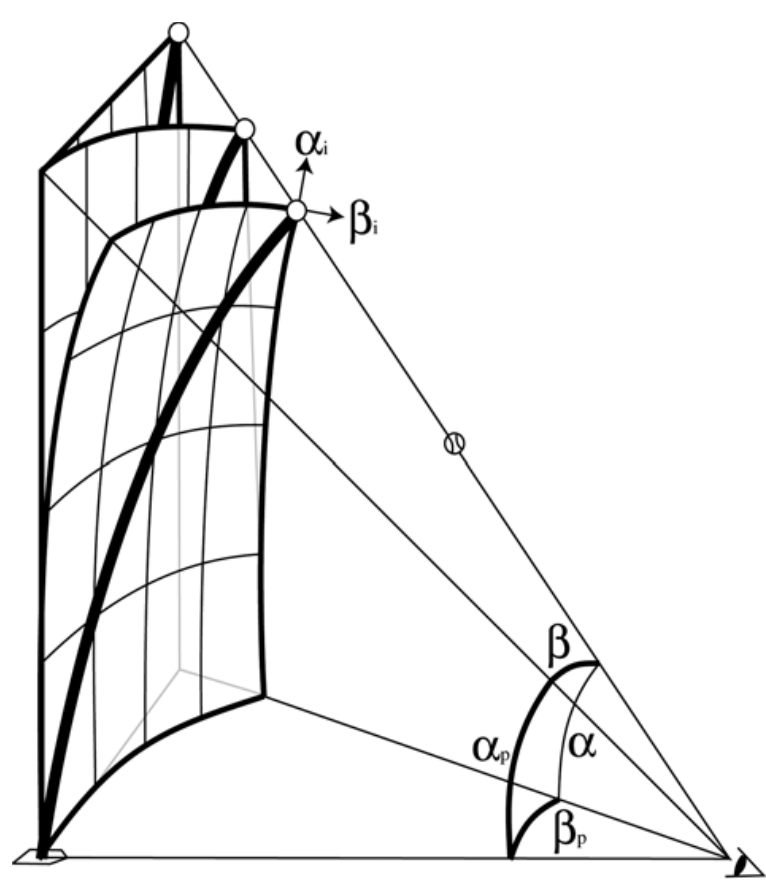

Figure 2. Geometry of the LOT model. Angled view of LOT with planar, cylindrical, and spherical projection geometry. Several ways of defining vertical and lateral optical angles are shown. First, $\alpha_{p}$ and $\beta_{p}$ indicate vertical and lateral angles of the ball, as measured within planes that intersect the point of origin (planar projection). Second, $\alpha_{i}$ and $\beta_{i}$ indicate vertical and lateral angles of the ball, as measured within planes that intersect the ball (cylindrical projection). Specifically, $\alpha$ and $\beta$ (spherical projection) are the ongoing sum of the instantaneous changes in vertical and lateral optical velocity, $\alpha_{i}$ and $\beta_{i}$. 
One example of the differences between our definition of $\alpha$ and $\beta$, above, and that of others can be seen from McLeod et al.'s (2001) previous definition. In that study, the ball was lofted very high, relative to fielder depth. Because these balls started out and landed so close to fielders, and were in the air for $2.9 \mathrm{sec}$ while traveling forward only $13.9 \mathrm{~m}$, very large vertical optical angles, $\alpha$, were achieved. Whereas McLeod et al. (2001) found that $\alpha$ reached angles of between about $60^{\circ}$ and $75^{\circ}$, in a replication of McLeod et al.'s (2001) conditions (described in Experiment 2), we found that $\alpha$ reached angles of between about $70^{\circ}$ and $90^{\circ}$. We suggest that this increase in optical visual angle, $\alpha$, can be explained by a difference in the way we define angles.

McLeod et al. (2001) defined $\alpha$ as the vertical angle relative to the horizon, whether or not the ball passes beyond the fielder. Thus $\alpha$ can never exceed $90^{\circ}$, and would rarely even approach it. If a fielder continued to bend his head back as a ball traveled directly overhead and beyond, this characterization would describe $\alpha$ as increasing up to $90^{\circ}$, then decreasing toward $0^{\circ}$. In contrast, when optical angles are defined as the sum of instantaneous changes along a spherical projection plane, the measured optical angle depends on the orientation of the fielder. If he continues to bend his head straight back, the lateral optical angle, $\beta$, will remain relatively constant, and the vertical optical angle, $\alpha$, can exceed $90^{\circ}$. If he turns his head sideways, the lateral optical angle, $\beta$, will sweep up and the vertical optical angle, $\alpha$, will remain fairly constant. These dramatic differences that depend on the orientation of the fielder are a hallmark of viewer-based geometry. Defining the vertical and lateral optical angles in this manner seems to solve some geometric ambiguities in previous work, and also clarifies what we consider to be the best way to define optical angles.

\section{The Recent McLeod et al. (2006) Model- Maintenance of $d \alpha / d t$ and $d \beta / d t$}

A new strategy for catching baseballs has been described by McLeod et al. (2003) and further described by McLeod et al. (2006). They presented a theory and simulated data that supported the idea that fielders catching baseballs headed to the side keep their gaze moving upward at a decreasing rate, thereby guaranteeing interception (McLeod et al., 2001, 2003, 2006). They also claimed, and showed, that fielders simultaneously move their gaze laterally, relative to the origin and at a constant rate.

\section{Fundamental Differences Between the LOT and Maintaining Constancy of $d \beta$ and Increasing $d \alpha$}

First, the LOT strategy specifies predictions in terms of the optical angles $\alpha$ and $\beta$. The McLeod strategy specifies predictions in terms of the derivatives (or velocities) of these angles, $d \alpha / d t$ and $d \beta / d t$. Second, the specificity of the descriptions is different. The LOT has a very specific prediction about the optical information- $\psi$, or the angle of the optical image of the ball off the ground, must be constantly maintained to result in an LOT (or the optical angles $\alpha$ and $\beta$ must change proportionally to achieve an LOT). This specificity brings with it a geometrical guarantee - that the fielder will be in the right spot just in time to catch the ball if this strategy is used.

In contrast, the generalized optical acceleration cancellation (GOAC) model first specifies that fielders must run so that vertical optical angle $\alpha$ increases. Although much of the evidence suggests that fielders do run so that $\alpha$ increases (McBeath et al., 1995; McLeod \& Dienes, 1996; McLeod et al., 2003, 2006), one of the disadvantages is that controlling the velocity of the vertical optical angle $\alpha$ in this way does not guarantee interception, which is geometrically guaranteed when fielders keep the tangent of $\alpha(\tan \alpha)$ increasing at a constant rate (or maintaining constant acceleration of the ball) (Chapman, 1968). At angles near $30^{\circ}$, $\alpha$ can be kept increasing at a constant rate to satisfy the requirement that $\tan \alpha$ increases at a constant rate, because $\alpha \approx \tan \alpha$ (i.e., the law of small angles). Between optical angles of $\sim 30-70$, the velocity of the vertical optical angle will decrease, almost regardless of what the fielder is doing. However, the way it does this depends on ball height and fielder position relative to the ball (McBeath, Shaffer, \& Sugar, 2002; McBeath et al., 1995; Shaffer \& McBeath, 2002). For instance, at angles approaching $60^{\circ}-90^{\circ}$, a constant rate of change in $\tan \alpha$ (i.e., constant acceleration) produces a change in $\alpha$ that slows down exponentially (Schneider et al., 1978; Shaffer $\&$ McBeath, 2002). Thus, the exact manner in which $\alpha$ must be controlled to guarantee interception may change on a trial-by-trial basis. The GOAC model then specifies that the fielder must also run in such a way as to maintain constancy of the velocity of the lateral angle (or in such a way that the lateral angle does not accelerate). The advantage to this is that there is no specific lateral strategy to be adopted; this allows for a range of possible lateral strategies to achieve interception.

Third, the new way the LOT is defined does not require maintenance of the optical ball trajectory relative to its origin. Defining $\alpha$ and $\beta$ as the ongoing sum of all instantaneous changes in vertical and lateral optical target position allows pursuers to keep their eyes on the target without having to maintain the optical target image relative to its origin, similar to other models of catching behavior (e.g., Michaels \& Oudejans, 1992; Oudejans et al., 1999; Peper, Bootsma, Mestre, \& Bakker, 1994). The GOAC model (and even our former definitions of LOT) uses the point of origin as a reference for all other data points.

Fourth, maintaining a 2-D LOT makes intercepting targets headed to the side computationally simpler. Although this alone may not mean that it is easier for the human perceptual system to detect, it is also consistent with fielders who report that it is easier to discern where a ball is headed when it is directed to their side rather than in the plane directly toward them (Shaffer \& McBeath, 1997). In contrast, GOAC specifies that two different optical angles are controlled differently, theoretically making the task of monitoring and changing optical information computationally more complex.

Finally, the LOT is based on direct measurements of where the fielder is looking (i.e., the optical trajectory) while he is running to intercept a target. McLeod et al. (e.g., McLeod et al., 2001, 2003) indirectly assess the op- 
tical trajectory by modeling the ball trajectory, and use the head of the fielder as an index of where they are looking, as do other models of ball catching behavior.

Experiment 1 was performed to test the domain generality of both GOAC and LOT strategies. Whereas both models have evidence that support that these strategies are used within baseball (McBeath et al., 1995; McLeod et al., 1996, 2003, 2006; Shaffer \& McBeath, 2002; Shaffer, McBeath, Roy, \& Krauchunas, 2003), testing their viability in Frisbee catching provides a good test of the robustness of the strategies across domains and with a target that moves in a different manner than a baseball. Although the movement of baseballs is not simple (e.g., Brancazio, 1985; Watts \& Bahill, 2000), it is fairly predictable. In contrast, Frisbees have a completely different flight pattern, and they may dramatically change speed and direction midflight. Experiment 1 then tests whether maintaining constancy of $d \delta / d t$ and keeping $d \alpha / d t$ decreasing explains Frisbee-catching behavior. Experiment 2 was performed to investigate whether the LOT and maintaining $d \alpha$ and $d \beta$ are tracking strategies not limited to baseball (i.e., are more generic tracking strategies). That is, perhaps both work because they depend on the characteristics of the flight of the targets. In this way, they might provide effective invariants for pursuit across many domains.

\section{EXPERIMENT 1}

\footnotetext{
Method

Participants. Four male Frisbee catchers (age range 18-36 years) participated in the experiment. Participants had between 5 and 20 years of experience catching Frisbees and had each played on competitive "Ultimate Frisbee" teams.

Procedure. Catchers stood approximately $20 \mathrm{~m}$ from the person throwing a standard 175-g Frisbee. We mounted a Supercircuits inline weather-resistant micro video camera (model no. PC75WR) on a bicycle helmet that catchers wore. The camera was wired to a battery pack and to a transmitter that the catcher wore on a pack around his waist. The signal from the camera was then transmitted to a receiver attached to a remote VCR, so that the VCR recorded the image or optical location of the Frisbee from the micro video camera.

Frisbees were launched at a variety of small angles (between $0.5^{\circ}$ and $\sim 2^{\circ}$ ), all off to the side of the catcher's initial position, at varying force from a distance of approximately 10 to $25 \mathrm{~m}$. Catchers ran between 5 and $20 \mathrm{~m}$ to catch the Frisbee, which was thrown a total of 86 times ( 20 per catcher, except for one catcher who had 26 trials). There were 75 trials in which the catcher kept the Frisbee within the field of view of the camera, the catcher eventually caught the Frisbee, and the Frisbee was not launched directly at the catcher; 4 times it was thrown in the same plane directly toward the catcher (i.e., there was no lateral offset); and 7 times the Frisbee was not caught. We recorded the ongoing instantaneous optical position of the center of the Frisbee relative to distant background markers each $1 / 30 \mathrm{sec}$. The ceiling and walls were textured so that there were enough distinct and clearly identifiable markers against which to code the Frisbee's position. The data were coded as pixel movements, then converted to degrees visual angle. This conversion produces very precise visual angles, provided the Frisbee is compared with background scenery that is distant and remains within a small visual angle of it. In this work, the comparison background scenery was always within $1^{\circ}$ visual angle of the ball, well within the law of small angles. We defined $\alpha$ and $\beta$, respectively, as the ongoing sums of all instantaneous vertical and lateral changes in the position of the Frisbee image, as defined previously and in Figure 2.
}

We separately analyzed the optical paths of the 15 trajectories on which the Frisbee, making a trajectory perturbation, began moving in a new direction and at a new speed from its initial direction and speed. Our analysis tested whether catchers used multiple LOT strategies, consistent with midflight changes in trajectory. The 15 trials, then, resulted in 30 separate portions ( 2 per trial) for which we tested multiple LOT strategies. On 7 of these, the Frisbee fell before being caught. The experimenters selected which trajectories had perturbations after the throw was made. During the coding stage, independent observers naive to the hypotheses of the experiment also separately identified trajectories with perturbations

The optical trajectory was measured by recording the Frisbee position for each video frame onto a transparency taped to the video monitor. The transparency was moved frame by frame on a video monitor to keep the background aligned. Visual angle was calibrated by converting the pixel height of the thrower on the video image to the actual visual angle of the thrower observed at the time of recording. The initial location of the Frisbee out of the thrower's hand was marked as the $(0,0)$ coordinate. The data were coded as pixel movement, then converted to degrees visual angle. This conversion produces very precise visual angles, provided the Frisbee is contrasted with distant background scenery and remains within a small visual angle of it. In this work, the comparison background scenery on the walls, ceiling, and floor was always within $0.5^{\circ}$ visual angle of the Frisbee, well within the region of the law of small angles. We also had static test trials in which we varied the angle of the camera and verified that any errors in the optical angle were insignificant.

\section{Results}

Analysis of LOTs and double LOTs. For each of the first 60 trials, we plotted the ongoing optical position of the Frisbee in terms of $\alpha$, the vertical visual angle, by $\beta$, the lateral visual angle, and determined the best-fit linear function to assess the variance accounted for by a straightline optical trajectory (i.e., an LOT). A linear function (representing a monotonically increasing LOT) accounted for a median of over $96 \%$ of variance in optical movement of the Frisbee, and at least $95 \%$ for each catcher. For 57 of the 60 trials, the correlation between $\alpha$ and $\beta$ was at least .95 . In order to test whether an LOT strategy was maintained to this threshold in a significant number of trials (vs. $<95 \%$ ), we performed a chi-square test. The chi-square test provided significant support for the LOT strategy $\left[\chi^{2}(1, N=60)=\right.$ 48.6, $p<.001]$. Eight representative optical Frisbee trajectories are shown in Figure 3. The data were coded in spherical coordinates as described earlier, then converted to the undistorted projection plane coordinates shown here. The view shown in Figure 3 is the view an onlooker would have with the video camera mounted on a helmet on his or her head as he or she ran to catch a Frisbee. Consistent with previous evidence (Oudejans et al., 1999), fielders fixated the ball as they ran to catch it, in line with the predictions of the LOT strategy. The plots show where the ball was fixated relative to the background scenery. The lines over each of the trajectories shown in the figure are estimates of the best-fit lines used to compute the $R^{2}$ values. They help illustrate that the trajectories remain close to straight lines. We also analyzed whether the Frisbee catcher's running speed and direction maintained proportional changes in the lateral and vertical optical angles to achieve an LOT as we have shown previously (Shaffer et al., 2003). Sixty regression analyses confirmed that changes in the vertical optical angle $\alpha$ significantly predicted changes in the lat- 


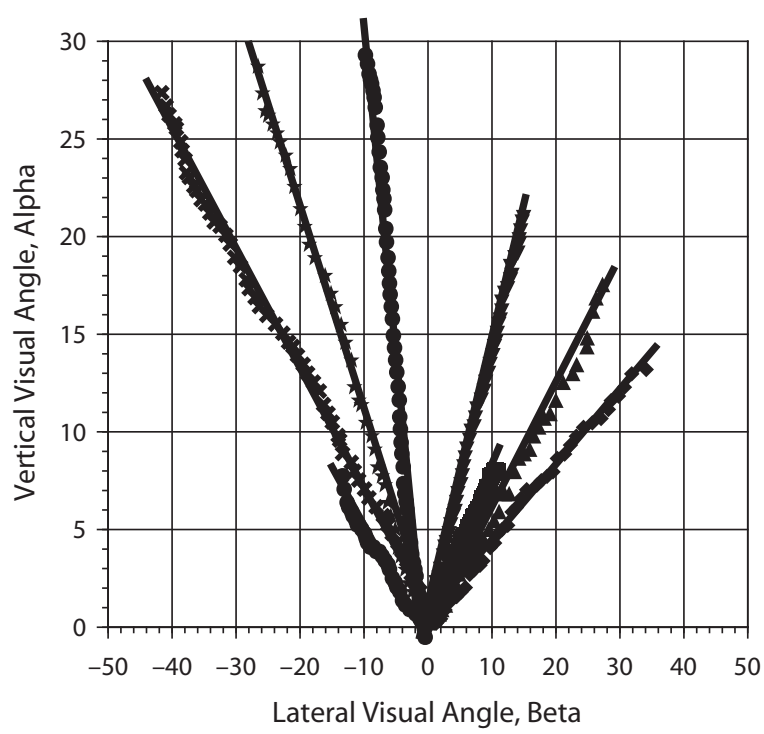

Figure 3. Sample optical Frisbee trajectories. The figure shows the optical trajectories for Frisbees on eight representative trials. Frisbee position is plotted as the lateral visual angle $\beta$ (in degrees), by the vertical visual angle $\alpha$ (in degrees) at each 1/30sec video frame. The coordinate 0,0 indicates the starting optical position of the fielder, and the highest symbol within each trajectory indicates the final optical position. The lines over each of the trajectories shown in the figure are estimates of the best-fit lines used to compute the $R^{2}$ values.

eral optical angle $\beta$ for all 60 trajectories, with $F$ s ranging from 51.43 to 77,950 (median $F$ value $=2,184$ ), all $p$ s $<$ .001 .

We separately analyzed the optical paths of the 15 trials where the Frisbee made a large perturbation during the trajectory and began moving in a new direction and at a new speed from its initial direction and speed. In 30 trials, catchers used multiple LOT strategies, consistent with midflight changes in the Frisbee trajectory. In these cases, a double LOT, or two straight lines, accounted for an average of over $95 \%$ of the variance. Additionally, before being caught, Frisbees can be chased while they hover for several seconds high in the air or just above the ground. If the Frisbee first went up in the catcher's field of view, then fell and was allowed to hover just above the ground before being caught, the catcher would be looking below the level from which it was first thrown (i.e., its origin), and would have to maintain an LOT that moved down rather than up. This is what happened for the second half of several double LOT trials. Three examples of double LOTs are shown in Figure 4, two of which have LOTs midflight in the downward direction. In these cases, the optical path of the Frisbee was moving in one direction and then suddenly began moving in a dramatically different direction. As Figure 4 shows, the fielders do not appear to abandon the LOT strategy, but simply to choose one LOT strategy before the large perturbation is made, then, after the perturbation, revise to another.

Analysis of $\boldsymbol{d \alpha} / \boldsymbol{d} \boldsymbol{t}$ and $\boldsymbol{d} \boldsymbol{\beta} / \boldsymbol{d t}$. In order to test the manner in which catchers controlled the velocity of the ver- tical optical angle, $d \alpha / d t$, when pursuing and catching Frisbees, we analyzed the same 60 Frisbee-catching trials. To do this, we plotted the change in $\alpha(d \alpha)$ against time and tested the significance of the correlation coefficient. The velocity of $\alpha(d \alpha / d t)$ was maintained at a constant rate (20 trials) as often as, at a significantly decreasing rate (28 trials), in increased $\left[\chi^{2}(1)=1.33, p>.1\right]$; in the other 12 trials, $d \alpha / d t$ increased. Over $53 \%$ of the correlation coefficient values are either zero or positive, and $r$ values formed a random distribution, ranging from -.90 to +.74 .

Analysis of $\boldsymbol{d} \boldsymbol{\beta} / \boldsymbol{d t}$. In order to test the McLeod et al. (2001) claim that fielders keep the velocity of the lateral optical angle $\delta(d \beta / d t)$ constant, we analyzed whether catchers kept $d \beta / d t$ constant significantly more often than they kept it increasing or decreasing. We plotted the velocity of $\beta(d \beta)$ against time and tested the significance of the correlation coefficient. The velocity of $\beta$ was kept significantly decreasing (19 trials) and significantly increasing (22 trials) as often as it was kept constant (19 trials) $\left[\chi^{2}(2)=0.30, p>.1\right]$. Almost $70 \%$ of the correlation coefficient values are significantly increasing or decreasing. Consistent with this, $r$ values formed a random distribution, ranging from -.87 to +.85 .

\section{Discussion}

Catchers maintained an LOT for the optical Frisbee trajectory in order to catch Frisbees. In order to do this, catchers maintained proportional changes in $\alpha$ and $\beta$ throughout each of the 60 trajectories. Because use of the LOT is not tied to the target's origin, multiple LOTs may be used if there are perturbations midflight that cause the catcher to change speed and direction (Shaffer et al., 2004). Whereas baseball trajectories are somewhat predictable once initiated - even though aerodynamic drag and ball spin cause them to deviate from perfect parabolic motion (Brancazio, 1985; Watts \& Bahill, 2000)_Frisbees may dramatically change direction and speed, depending on such factors as how they are thrown, gusts of wind, and the Frisbee angle during flight (Bloomfield, 1999).

Keeping $d \alpha / d t$ increasing at a decreasing rate works no better for catching Frisbees than keeping it increasing at a constant rate does. Experiment 1 also showed that keeping $d \beta / d t$ constant is not a strategy used to catch Frisbees that accounts for lateral behavior of catchers, largely because of the random way in which it is maintained.

In order to compare these two strategies graphically within trials, we have plotted one trial from each of the four participants in Figure 5. The left panels are plots of $\alpha$ and $\beta$ (i.e., an LOT), the middle panels are plots of $d \beta /$ $d t$, and the right panels are plots of $d \alpha / d t$, for the same trials. Although the optical trajectories remain very close to straight lines, $d \beta / d t$ decreases slightly, then increases at slightly different rates (as one looks at the middle panel of graphs from top to bottom); $d \alpha / d t$ does the same. The only constant across the $d \alpha / d t$ and $d \beta / d t$ plots is that the pattern in $d \alpha / d t$ is matched by the pattern in $d \beta / d t$, reflective of keeping the changes in $\alpha$ proportional to the changes in $\beta$ to maintain an LOT (McBeath et al., 1995; Shaffer et al., 2003). 

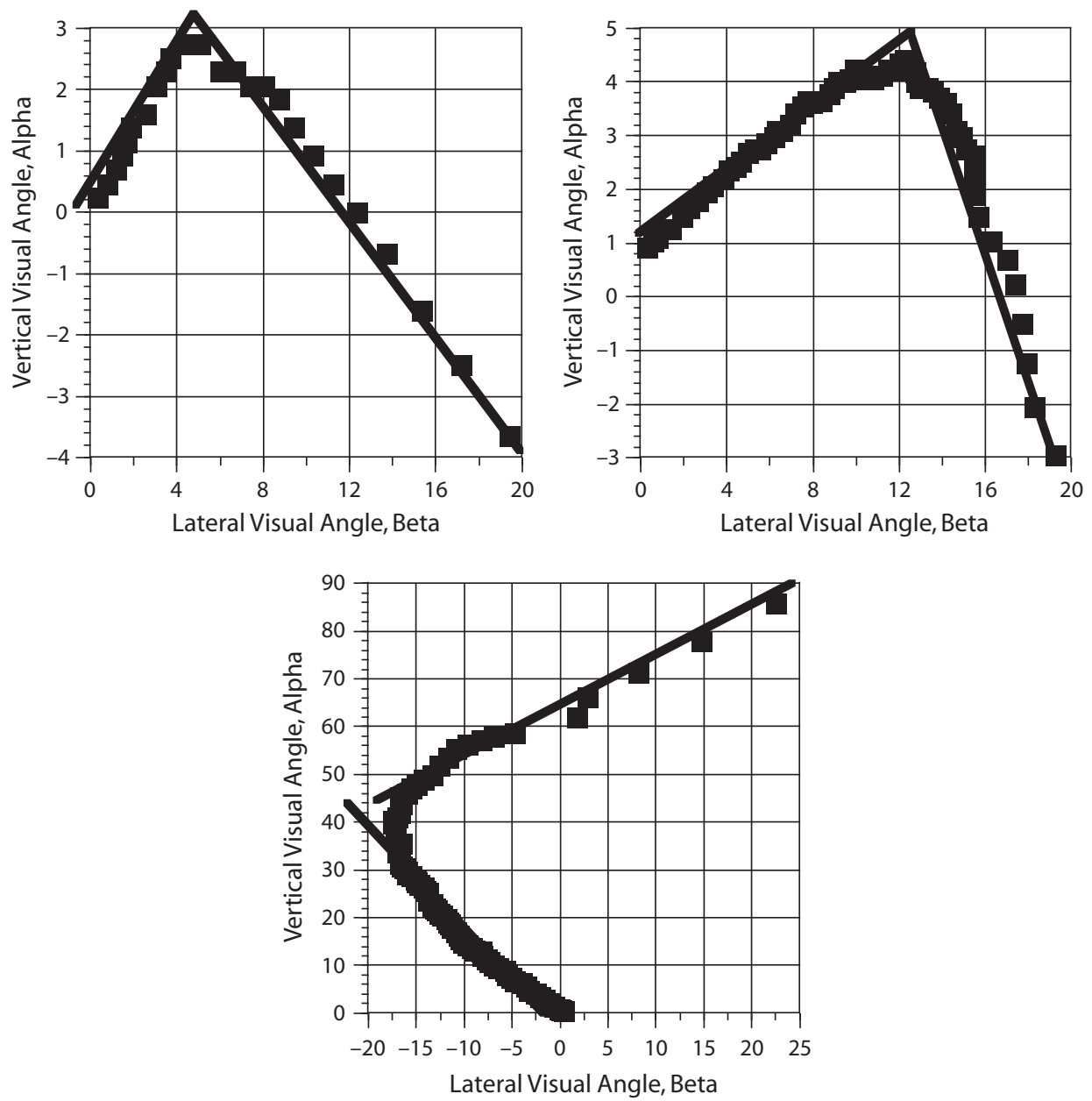

Figure 4. Double LOT trajectories. Three representative optical trajectories of Frisbees are shown in which the Frisbee was deliberately thrown to make a large perturbation in its trajectory. Observed Frisbee position is plotted as the lateral visual angle $\beta$ (in degrees), by the vertical visual angle $\alpha$ (in degrees) at each $1 / 30$-sec frame. The lines over each of the trajectories shown in the figure are estimates of the best-fit lines used to compute the $\boldsymbol{R}^{2}$ values. In these cases, when the initial control conditions started to break down, the catcher substituted in a replacement LOT and converged to interception.

It should be noted that although we have shown that GOAC is not viable for Frisbee-catching behavior, McLeod et al. (2001) made no claims that GOAC should be used outside the domain of baseball. Thus, though the findings here show the limitations of the domain generality of GOAC, they do nothing to dismiss GOAC as a viable model of baseball-catching behavior.

\section{EXPERIMENT 2}

Experiment 2 was performed for two reasons: First, we felt it was necessary to test whether an LOT would be maintained for fly balls as it was for Frisbees. There is already abundant evidence that the LOT is maintained by tens of different fielders across hundreds of trials of catching fly balls (e.g., McBeath et al., 1995; Shaffer \& McBeath, 2002; Shaffer et al., 2003), so we limited our analysis in this experiment to two fielders and 20 trials of catching fly balls. Second, although $d \alpha / d t$ broke down for Frisbee catching, it is widely agreed that $d \alpha / d t$ decreases for catching typical to very high fly balls, even though the way $d \alpha /$ $d t$ is controlled is not straightforward (as mentioned in the introduction to Experiment 1). Thus, because controlling $d \alpha / d t$ so that it decreases works for catching fly balls, $d \beta / d t$ may also work, even though both break down when applied to Frisbee catching. That is, perhaps both work because they depend on the characteristics of the flight of the targets. In this way, they might provide an effective alternative to the LOT strategy for catching fly balls, even though controlling them in the way specified by McLeod et al. (2001) is not effective in Frisbee catching.

\section{Method}

Participants. Two good recreational outfielders, ages 19 and 31 years, were each presented with 10 trials of catching fly balls.

Procedure. We mounted a Supercircuits inline weather-resistant micro video camera (Model PC75WR) on a bicycle helmet, which 

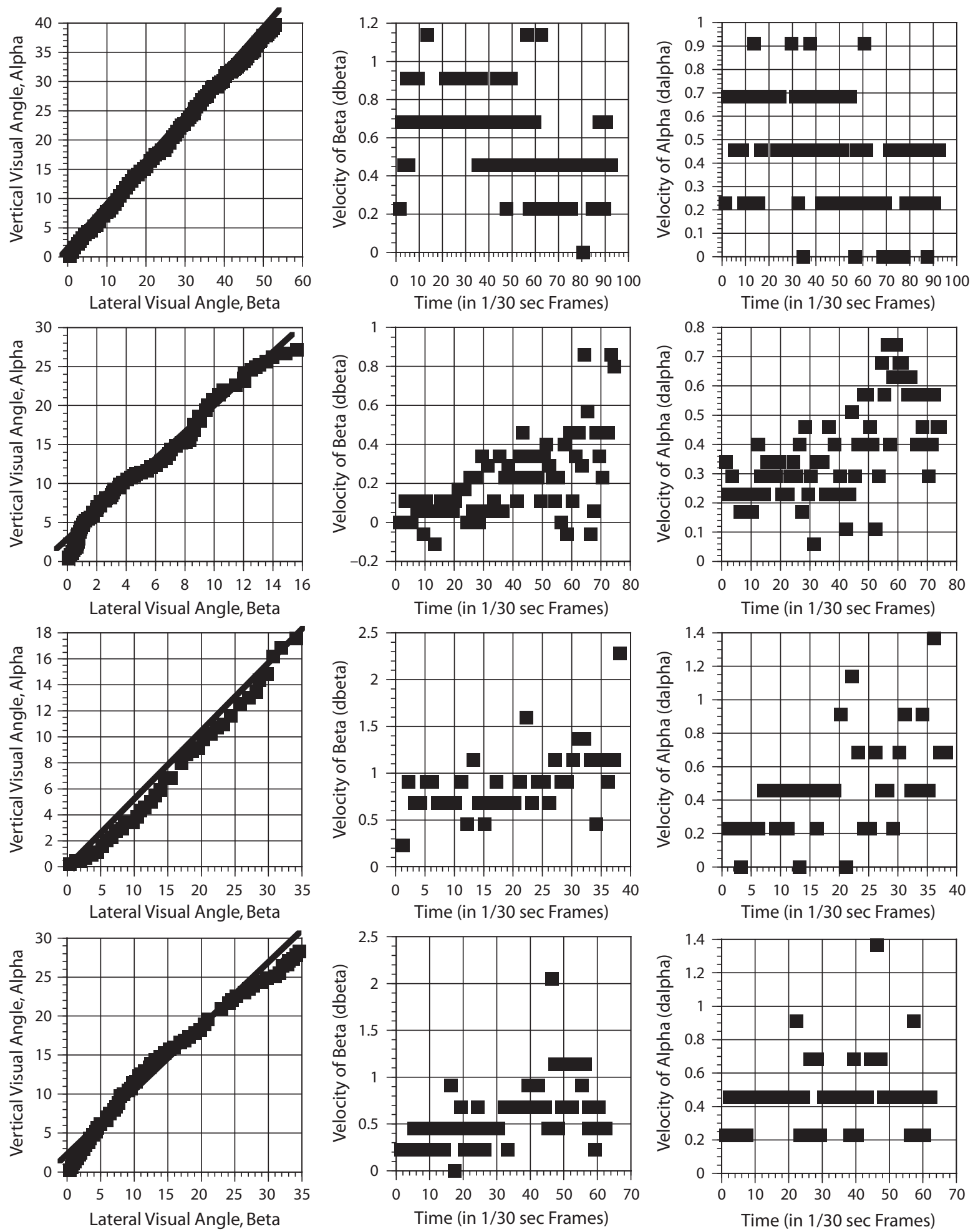

Figure 5. Maintaining an LOT versus $d \alpha / d t$ and $d \beta / d t$. The left panels show the vertical optical ball angle, $\alpha$, plotted against the lateral optical ball angle, $\beta$, for one trial for each of the four catchers, respectively. The lines over each of the trajectories shown in the figure are estimates of the best-fit lines used to compute the $\boldsymbol{R}^{2}$ values. The middle panels show the velocity of the lateral optical ball angle $(d \beta / d t)$, for the same trials plotted in the corresponding left panel. The right panels show the velocity of the vertical optical ball angle $(d a / d t)$, for the same trials plotted in the corresponding left and middle panels. 
the fielders wore. The camera was wired to a battery pack and to a transmitter that the fielders wore on a pack around their waists. The signal from the camera was then transmitted to a receiver attached to a remote Cannon GL1 digital video camcorder, which recorded the image from the micro video camera. We coded optical ball position from the ongoing video image. The ongoing vertical and lateral optical angles were obtained as they were in Experiment 1.

In order to test whether fielders use an LOT and maintain $d \alpha /$ $d t$ and $d \beta / d t$ as McLeod et al. (2001) specified, we used a setup similar to that in McLeod et al.'s (2001) study. ${ }^{2}$ We did this for two reasons. First, as McLeod et al. stated in their article, the positions provide a wide range of ball trajectories, from easy catches to very difficult catches, for which both the strategies investigated in this article should work. Second, the LOT as we have defined it has not been tested under these more extreme conditions of "shallow" fly balls (closer to "pop-ups" in baseball) launched from a fairly close distance; see McLeod et al. (2001) for further details.

\section{Results}

Analysis of maintaining an LOT. A linear optical trajectory accounted for an average of over $96 \%$ of the variance, ranging from $91 \%$ to $99 \%$, over all 20 trials examined, and with no position average less than $95 \%$. We again analyzed whether the fielder's running speed and direction maintained proportional changes in the lateral and vertical optical angles to achieve an LOT. Twenty regression analyses confirmed that changes in the vertical optical angle $\alpha$ significantly predicted changes in the lateral optical angle $\beta$ for all 20 trajectories, with $F_{\text {s }}$ ranging from 135.38 to $6,054.89$ (median $F$ value $=1,604.08$ ), all $p$ s $<.001$. Figure 6 shows representative optical trajectories plotted as $\alpha$ by $\beta$. These trajectories have been plotted until the last $\frac{1}{3} \mathrm{sec}$ before the ball hits the fielder's glove. The plots show where the ball was located relative to the background scenery as fielders fixated the ball, and represent undistorted spherical coordinates converted to projection plane coordinates seen in the plots. As can be seen, the optical trajectories remain close to straight lines, as predicted by the LOT strategy. In spite of the fact that the LOT accounted for over $96 \%$ of the variance in ball movement, there is a pattern of slight curvature near the ends of the trajectories. To our knowledge, this curvature only appears in the extreme case of "pop-up" types of balls in baseball or balls that are eventually not caught (e.g., Shaffer \& McBeath, 2002). In Figure 7, we have plotted four representative trajectories (two from McLeod et al.'s [2001] Position 1 and two from Position 2). In our previous work (and in Figure 6), we elected to plot trajectories only up until the last $\frac{1}{3}$ to $\frac{1}{2} \mathrm{sec}$. However, in Figure 7, we plot all of the data points up until the ball is caught, to demonstrate the effect of the change in optical ball size. We include horizontal "optical ball size" bars at each optical location of the ball corresponding to the optical ball size in degrees visual angle. Figure 7 shows how the deviation from linearity, which can be seen toward the ends of the trajectories plotted on the left of the graph, is within the range of the increase in optical size of the approaching ball, and in the direction expected when the fielder's head is offset due to catching the ball with the preferred glove handedness. If one considers the ambiguity in linearity introduced by the increase in the optical size of the ball, the curvature near the ends of the trajectories remains in the range of the optical ball size.
Analysis of $\boldsymbol{d} \boldsymbol{\alpha} / \boldsymbol{d} \boldsymbol{t}$ and $\boldsymbol{d} \boldsymbol{\beta} / \boldsymbol{d} \boldsymbol{t}$. Consistent with all other studies of fielders catching fly balls, $d \alpha / d t$ significantly decreased in all but one of the 20 trials $\left[\chi^{2}(1)=16.2, p<\right.$ $.001]$. In order to test the GOAC claim that fielders keep the velocity of the lateral optical angle $\beta(d \beta / d t)$ constant, we again analyzed whether fielders kept $\mathrm{d} \beta / d t$ constant more often than they kept it increasing or decreasing. We again plotted the velocity of $\beta(d \beta / d t)$ against time and tested the significance of the correlation coefficient. The velocity of $\beta$ was kept significantly decreasing (7 trials) and significantly increasing ( 3 trials) as often as it was kept constant (10 trials) $\left[\chi^{2}(2)=3.7, p>.5\right]$.

\section{Discussion}

In Experiment 2, an LOT was maintained, accounting for over $96 \%$ of the variance in optical ball movement for shorter "pop-up" fly balls in baseball. Even when the ball trajectory was analyzed until the moment of the catch, it was maintained in a monotonically increasing LOT, within $1 S D$ of optical ball size. This demonstrates the generality of the LOT heuristic across different types of fly balls in baseball and across catching domains and targets. Maintenance of $d \beta / d t$ for baseball-type "pop-ups" was random. In predicting whether the ball would be caught, maintaining $d \beta / d t$ constant was no better than maintaining it at a significantly increasing or decreasing rate. Thus, GOAC does not appear to work for catching high fly balls at a short range.

Impact on predictions of LOT and GOAC strategies of high fly balls launched from a short distance. Empirical evidence supports both LOT and GOAC strategies as being effective solutions for running to catch fly

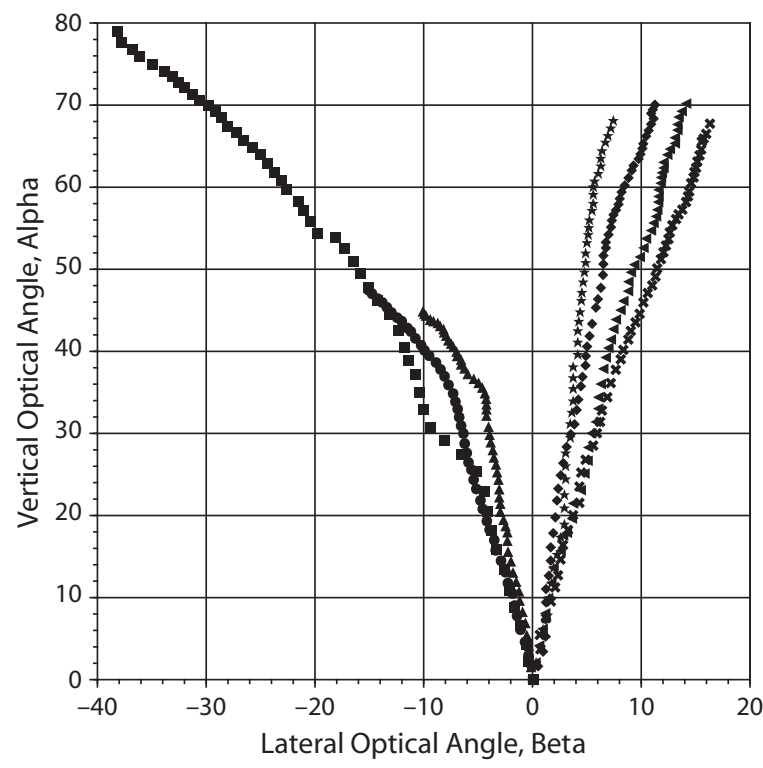

Figure 6. Sample optical baseball trajectories. The figure shows the optical trajectories for baseball for several representative trials. Ball position is plotted as the lateral visual angle, $\beta$ (in degrees), by the vertical visual angle, $\alpha$ (in degrees), at each 1/30sec video frame. These trajectories were plotted up until the last $1 / 3$ sec before the ball hits the fielder's glove. 

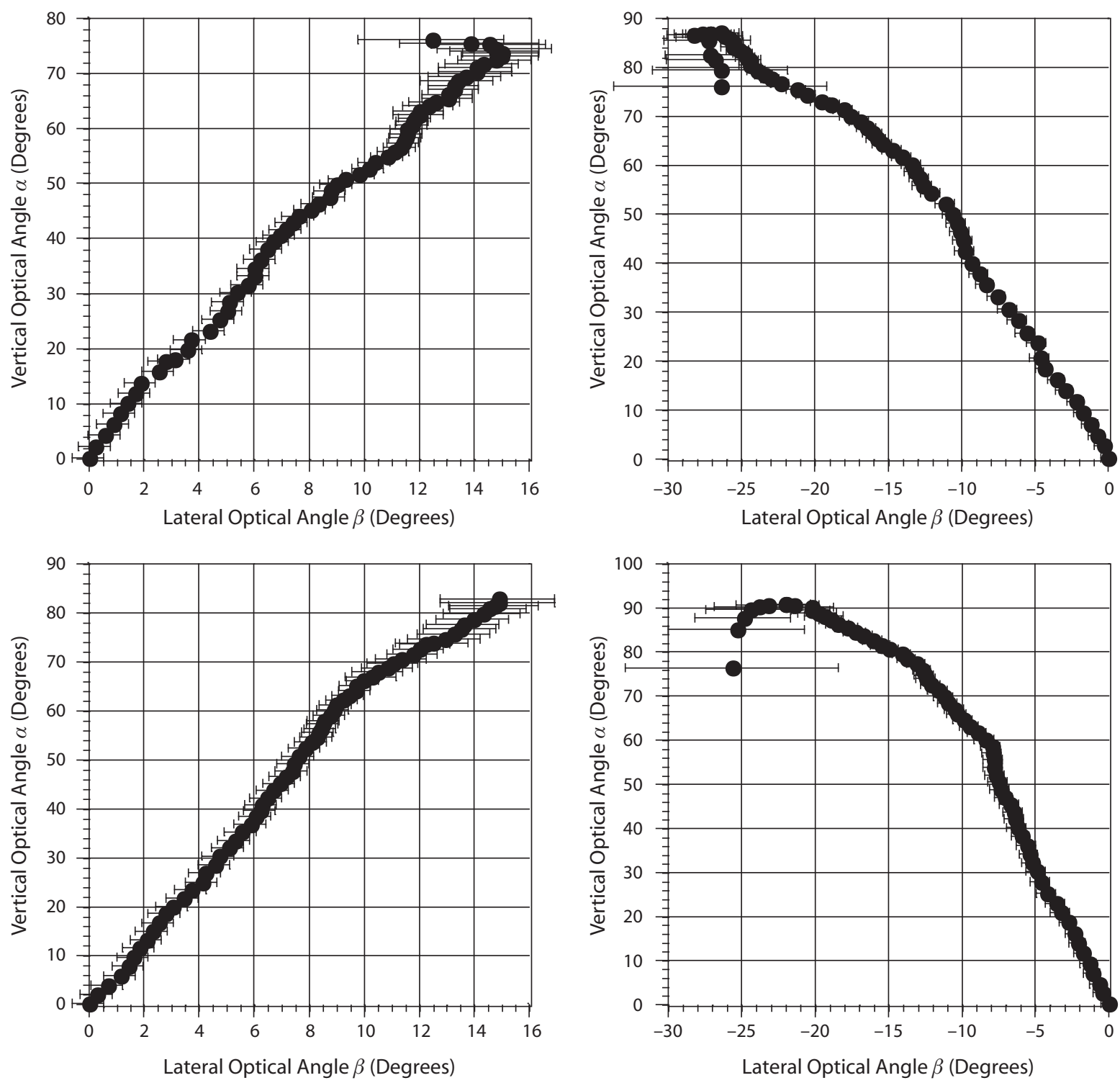

Figure 7. Fielders maintain an LOT until the ball is caught. Shown are the vertical and lateral optical angles, $\alpha$ and $\beta$, for four typical examples of optical ball position at 1/30-sec intervals. The two trajectories on the left (top and bottom) are from Position 2 , where the fielder starts $3 \mathrm{~m}$ in front of and $\mathbf{2} \mathrm{m}$ to the left of where ball lands. The two on the right are from Position 1, where the fielder starts $4 \mathrm{~m}$ in front of and $3 \mathrm{~m}$ to right of where the ball lands. The lateral optical ball size bars indicate the optical size of the ball.

balls characteristic of more prototypical fly balls in terms of height and distance (McBeath et al., 1995; McLeod et al., 2006; Shaffer et al., 2003). Here, we tested a special case of fly balls launched very high from a very short distance. Fielders were required to run only a few steps and direct their angle of gaze higher than for more typical fly balls. The result of this optically is that the vertical optical angle $\alpha$ will be great, in some cases approaching $90^{\circ}$ (see Figure 6), and that the lateral visual angle $\beta$ will be much larger than more typical fly balls (in Figure $6, \beta$ s are between $10^{\circ}$ and $40^{\circ}$, whereas in McBeath et al., 1995 , they are between $3^{\circ}$ and $8^{\circ}$ ). This is due to how close the fielder is to the ball to begin with and how close the fielder is to the eventual destination of the ball. This, in turn, means that slight movements of the head will lead to large changes in $\beta$.

These factors probably played roles in how the changes in $\beta$ (or $d \beta / d t$ ) were random and not constant, as GOAC predicts. Recall from above that $d \alpha / d t$ significantly decreased in all but one trial, whereas $d \beta / d t$ increased for 3 , decreased for 7 , and remained constant for 10 . For instance, a fielder who overruns the eventual landing destination by a little and moves back will have a $\beta$ that changes in the opposite direction during the ball's flight. Someone who approaches the ball more slowly and hurries in the final few steps could very likely have a $\beta$ that increases dramatically toward the end of the ball's flight. 
The LOT predicts that fielders will keep the ball moving in a straight line and do this by maintaining proportional changes in $\alpha$ and $\beta$, with a possible slight lag for changes in $\beta$ (Shaffer et al., 2003). Even though the LOT accounts for over $96 \%$ of the variance, the findings that $d \beta / d t$ decreased in 7 trials, increased in 3 , and remained constant in 10 are inconsistent with the LOT's prediction. Two reasons can explain why this occurs, and why it is not inconsistent with using an LOT. First, the ideal obeisance of LOT would be a perfectly straight line on which changes in both angles were perfectly proportional. However, neither LOT nor any other model has ever accounted for $100 \%$ of the variance in ball movement. We have consistently chosen a criterion of $95 \%$ to correspond to the $p<.05$ level of significance typically used in the field of psychology. This means that optical trajectories will remain close to, but not directly on top of, straight lines. In turn, $d \alpha / d t$ and $d \beta / d t$ will rarely be exactly proportional to one another. Second, when one takes the derivative of the angle, or of any value, there will typically be more variance in the derived values. Instead of framing proportional changes in terms of velocities, we have always expressed them in terms of plots of $\alpha$ and $\beta$ as functions of time, to see whether they were changing together. To test this, we correlated $\alpha$ as a function of time and $\beta$ as a function of time. We then compared correlations to see whether the correlations of $\alpha$ versus time and $\beta$ versus time for each trial were in the same direction (positive or negative). Consistent with the LOT strategy, proportional changes in $\alpha$ and $\beta$ were made in a statistically significant number of the trials $\left[\chi^{2}(1)=7.2, p<.005\right]$. This result may seem inconsistent with the analyses of $d \alpha / d t$ and $d \beta / d t$ in Experiment 2, but recall that in the analyses of Experiment 2 we were testing for constancy versus significant increases or decreases in those analyses; thus, $\alpha$ versus time could have $\alpha$ correlation of -.40 and $\beta$ versus time a correlation of -.30 and $\alpha$ might be significantly decreasing, whereas if $\beta$ were not significantly decreasing it would be categorized as statistically constant. So this can explain some of the seeming inconsistencies in the analyses of Experiment 2, and whether an LOT was being used.

It is the case, however, that under ideal obeisance of LOT one would expect the change in one angle to follow and match the change in the other. That is not occurring as consistently in the present work as is shown in the tops of the trajectories in Figure 6 that show that, as $\alpha$ is decreasing, $\beta$ is sometimes remaining constant, or it is decreasing with $\alpha$ but not at the same rate, resulting in the outward curvature.

In sum, it appears that the high "pop-up" type of balls used in the present work involve issues concerning the maintenance of consistent patterns of velocity of the lateral angle $\beta$ for LOT and GOAC. We discuss other factors that could contribute to the outward curvature seen in many of the trajectories in Figure 6 in the following section.

Factors contributing to deviations from linearity toward the ends of trajectories. As mentioned in the previous section, there is some curvature toward the ends of the ball trajectories in Experiment 2, which is more pronounced when optical ball position is recorded until the moment of the catch (in Figure 7). Experiment 2 also shows that even for the extreme types of fly balls that were tested, the LOT is maintained up until the moment the ball is caught, within $1 S D$ of optical ball size. This increase in optical ball size would lead to different optical trajectories, depending on whether linearity was being maintained for the center of the ball or for an edge. Optical ball size can also serve as a metric with which to evaluate optical curvature. In short, for these types of fly balls, optical curvature appears to have virtually always remained smaller than a few ball diameters. This is consistent with evidence from other sources that other spatial cues in addition to the optical trajectory become available and are presumably used during the final phase of interception (Regan, 1997; Regan \& Gray, 2001). The curvature also generally only occurred on those trials in which the fielder caught the ball with the left hand while running to the left. This is consistent with endpoint curvature due to the offset of the head and extended glove-hand. When fielders caught the ball while running to the right, they tended to hold their gloved hands closer to their heads. This is consistent with the absence of endpoint curvature due to the lack of offset between the head and gloved hand. When these geometric issues are considered, the optical trajectories are remarkably linear even until the point at which the ball hits the fielder's glove.

A second factor contributing to deviations from optical linearity toward the ends of the trajectories is glove handedness, particularly when comparing balls headed toward the gloved versus the ungloved side of the fielder. The impact of glove handedness may be even more salient in cases in which the fielder needs to move only a few steps, as in Experiment 2. Fielders typically position their bodies and extend their gloved hand toward the side from which a ball approaches. Thus, right- and left-gloved fielders who pursue identical fly balls will typically converge to points several feet apart. Because the player only needs to take a few steps in these pop-up experiments to reach the destination point, a lateral offset of an arm's length can be a substantial proportion of the fielder's overall movement from start to stop. We found that the occurrence of optical curvature of the ball is consistent with this handedness offset. Handedness typically produced the expected deviation from optical linearity beginning about $\frac{2}{3}$ to $\frac{1}{2} \mathrm{sec}$ before interception.

A third factor that could contribute to a systematic deviation from linearity of the optical trajectory toward the very ends of the trajectories is distortion of 3-D space that occurs across large optical angles. Given the occurrence of spatial distortions as great as $20 \%$ in large-angle phenomena like that of the moon illusion, the very definition of a straight line may come into question over these large optical angles. One popularly cited explanation of the moon illusion is that because the sky is perceived as a flattened dome, an object that moves above us will appear to have traveled more slowly and over a shorter distance than will the same extent of optical movement close to the horizon. If vertical and horizontal distances are distorted differently with elevation, a linear path would likely be similarly distorted. Distortions of similar size in real-world space 
have been demonstrated in judgments of hill slope (Proffitt, Bhalla, Gossweiler, \& Midgett, 1995) and estimates of lateral angles and distances in depth (Cuijpers, Kappers, \& Koenderink, 2001; Loomis \& Philbeck, 1999; Todd, Oomes, Koenderink, \& Kappers, 2001). In short, it seems quite likely that perception of space and location in the skyward direction involves distortions that are at least of the order as the slight curvatures in optical trajectories that were found in Experiment 2.

\section{GENERAL DISCUSSION}

McLeod et al. (2006) showed that both LOT and GOAC were good approximations for a modeled real fielder catching fly balls. Our work has shown that the LOT is used effectively by humans catching baseballs headed off to the side and chasing uncatchable balls, dogs catching Frisbees, and robots moving to intercept targets (McBeath et al., 1995, 1996; McBeath, Sugar, \& Shaffer, 2001; Shaffer et al., 2004; Shaffer \& McBeath, 2002; Sugar \& McBeath, 2001). The present work extends the previous work by showing that the LOT is also used by humans catching Frisbees. This, along with much previous work, demonstrates the domain-generality of the LOT heuristic. The present work has also shown that a double LOT-something that dogs use when catching Frisbees deliberately thrown to dramatically change direction and speed midflight - is used when humans catch Frisbees. The dogs used two different LOTs that both monotonically increased along optical straight lines (Shaffer et al., 2004). Here, in several cases, the second half of the double LOT moved downward because a Frisbee drops gently to the ground, allowing the catcher to catch it before it hits the ground. We also showed that GOAC was not extended to the domain of Frisbee catching. This does not show that GOAC is wrong, simply that it is domain-specific to more typical fly balls in baseball or cricket, as McLeod et al. (2006) theorized it should be.

In the extreme case of pop-ups in baseball, control of $d \beta / d t$ was random, not constant as predicted by GOAC. In contrast, a straight line accounted for over $96 \%$ of the variance in optical ball movement, as predicted by the LOT strategy. Here we have also shown that the LOT strategy is consistent with use of nonideal, real-world participants, optical ball size near terminus, coupled with a combination of individual differences in aggressiveness and handedness (McBeath, Shaffer, \& Sugar, 2002; McBeath et al., 2001). However, maintenance of proportional changes in the vertical and lateral angles was not maintained as consistently as predicted by LOT. We explained some of the reasons why GOAC may not work and why ideal obeisance of the LOT strategy may not be maintained in this extreme case.

The findings with humans catching Frisbees further support the generality of viewer-based navigational strategies and control heuristics (Cutting, Springer, Braren, \& Johnson, 1992; Cutting \& Wang, 2000; Shaffer et al., 2004; Royden \& Hildreth, 1996). Behavior consistent with maintaining spatiotemporal constancy between a pursuer and a moving target is found in many domains. For example, both aircraft and boat pilots can produce a collision course with another moving craft by maintaining an angle of bearing that remains constant relative to the image of the other craft (Pollack et al., 1995). Also, research shows that predator-prey characteristics of bats, birds, tethered flies, houseflies, and dragonflies are consistent with maintaining optical angle constancy between those predators and their prey (Collett \& Land, 1975; Jablonski, 1999; Olberg et al., 2000; Simmons, Fenton, \& O'Farrell, 1979). Finally, our work with automated mobile robots confirms the viability of viewer-based strategies, such as the LOT, for interception of moving projectiles (Sugar \& McBeath, 2001).

Interception of targets is evolutionarily important across species. Humans probably developed interceptive abilities consistent with the LOT strategy that are viewer-based; that are independent of the trajectory of a target; that are computationally simple; that allow the pursuer to make instantaneous changes in speed and direction before interception occurs; and that are not tied to the target origin. It is equivalent to maintaining a constant bearing angle between the pursuer and target, as is performed in many other domains and by different species. This makes the LOT appealing as a universal tracking heuristic that may have evolved from strategies used in other domains and by other species.

\section{AUTHOR NOTE}

S.M.K. is no longer at Saint Anselm College. Correspondence concerning this article and requests for materials should be addressed to D. M. Shaffer, Department of Psychology, Ohio State University, Mansfield, OH 44906 (e-mail: shaffer.247@osu.edu).

\section{REFERENCES}

Baud-Bovy, G., \& Viviani, P. (1998). Pointing to kinesthetic targets in space. Journal of Neuroscience, 18, 1528-1545.

Bloomfield, L. A. (1999). The flight of the Frisbee. Scientific American, 280, 132.

BranCAZIO, P. J. (1985). Looking into Chapman's homer: The physics of judging a fly ball. American Journal of Physics, 53, 849-855.

Chapman, S. (1968). Catching a baseball. American Journal of Physics, 36, 868-870.

Colby, C. L., \& Duhamel, J.-R. (1996). Spatial representations for action in parietal cortex. Cognitive Brain Research, 5, 105-115.

Colby, C. L., Duhamel, J.-R., \& Goldberg, M. E. (1995). Oculocentric spatial representation in parietal cortex. Cerebral Cortex, 5, 470-481.

Collett, T. S., \& LAND, M. F. (1975). Visual control of flight behaviour in the hoverfly, Syritta pipiens L. Journal of Comparative Physiology, 99, 1-66.

Cuijpers, R. H., Kappers, A. M. L., \& Koenderink, J. J. (2001). On the role of external reference frames on visual judgements of parallelity. Acta Psychologica, 108, 283-302.

Cutting, J. E., Springer, K., Braren, P. A., \& Johnson, S. H. (1992). Wayfinding on foot from information in retinal, not optical, flow. Journal of Experimental Psychology: General, 121, 41-72.

CutTing, J. E., \& WANG, R. F. (2000). Heading judgments in minimal environments: The value of a heuristic when invariants are rare. Perception \& Psychophysics, 62, 1146-1159.

DanNemiller, J. L., Babler, T. G., \& Babler, B. L. (1996). On catching fly balls. Science, 273, 256-257.

GiBson, J. J. (1979). The ecological approach to visual perception. Boston: Houghton Mifflin.

JABLONSKI, P. G. (1999). A rare predator exploits prey escape behavior: The role of tail-fanning and plumage contrast in foraging of the painted redstart (Myioborus pictus). Behavioral Ecology, 10, 7-14.

LoOMIs, J. M., \& PHILBECK, J. W. (1999). Is the anisotropy of perceived 3-D shape invariant across scale? Perception \& Psychophysics, 61, 397-402.

McBeath, M. K., Shaffer, D. M., \& Kaiser, M. K. (1995). How base- 
ball outfielders determine where to run to catch fly balls. Science, 268, 569-573.

McBeath, M. K., Shaffer, D. M., \& Kaiser, M. K. (1996). On catching fly balls. Science, 273, 258-260.

McBeath, M. K., Shaffer, D. M., \& Sugar, T. G. (2002). Catching baseball pop flies: Individual differences in aggressiveness and handedness. Paper presented at the 43rd Annual Meeting of the Psychonomic Society, Kansas City, MO.

McBeath, M. K., Sugar, T. G., Morgan, S. E., Oberle, C. D., Mundhra, K., \& Suluh, A. (2002, May). Human and robotic catching of dropped balls and balloons: Fielders still try to make the image of the projectile rise. Paper presented at the 2nd Annual Meeting of the Vision Sciences Society, Sarasota, FL.

McBeath, M. K., Sugar, T. G., \& Shaffer, D. M. (2001, May). Comparison of active versus passive ball catching control algorithms using robotic simulations. Paper presented at the 1st Annual Meeting of the Vision Sciences Society, Sarasota, FL.

McLeod, P., \& Dienes, Z. (1996). Do fielders know where to go to catch the ball or only how to get there? Journal of Experimental Psychology: Human Perception \& Performance, 22, 531-543.

McLeod, P., Reed, N., \& Dienes, Z. (2001). Toward a unified fielder theory: What we do not yet know about how people run to catch a ball. Journal of Experimental Psychology: Human Perception \& Performance, 27, 1347-1355.

McLeod, P., Reed, N., \& Dienes, Z. (2003). How fielders arrive in time to catch the ball. Nature, 426, 244-245.

McLeod, P., ReEd, N., \& Dienes, Z. (2006). The generalized optic acceleration cancellation theory of catching. Journal of Experimental Psychology: Human Perception \& Performance, 32, 139-148.

Michaels, C. F., \& Oudejans, R. R. D. (1992). The optics and actions of catching fly balls: Zeroing out optical acceleration. Ecological Psychology, 4, 199-222.

Olberg, R. M., Worthington, A. H., \& Venator, K. R. (2000). Prey pursuit and interception in dragonflies. Journal of Comparative Physiology, 186, 155-162.

Oudejans, R. R. D., Michaels, C. F., Bakker, F. C., \& Davids, K. (1999). Shedding some light on catching in the dark: Perceptual mechanisms for catching fly balls. Journal of Experimental Psychology: Human Perception \& Performance, 25, 531-542.

Peper, L., Bootsma, R. J., Mestre, D. R., \& Bakker, F. C. (1994). Catching balls: How to get the hand to the right place at the right time. Journal of Experimental Psychology: Human Perception \& Performance, 20, 591-612.

Pollack, H. N., Adair, R. K., Chodosh, L. A., Lifson, L. E., Tabin, C., JaCobs, R. A., McBeath, M. K., ET AL. (1995). Play ball! [Letters and response related to McBeath et al., 1995]. Science, 268, 1681-1685.

Proffitt, D. R., Bhalla, M., Gossweiler, R., \& Midgett, J. (1995). Perceiving geographical slant. Psychonomic Bulletin \& Review, 2, 409-428.

Regan, D. (1997). Visual factors in hitting and catching. Journal of Sport Sciences, 15, 533-558.

Regan, D., \& GRAY, R. (2001). Hitting what one wants to hit and missing what one wants to miss. Vision Research, 41, 3321-3329.

Royden, C. S., \& HiLdreth, E. C. (1996). Human heading judgments in the presence of moving objects. Perception \& Psychophysics, 58, 836-856.

Schneider, B., Ehrlich, D. J., Stein, R., Flaum, M., \& Mangel, S. (1978). Changes in the apparent lengths of lines as a function of degree of retinal eccentricity. Perception, 7, 215-223.

Shaffer, D. M., Krauchunas, S. M., Eddy, M., \& McBeath, M. K. (2004). How dogs navigate to catch Frisbees. Psychological Science, 15, 437-441.

Shaffer, D. M., \& McBeath, M. K. (1997). [Responses concerning catching fly balls hit off to the side and directly toward an outfielder]. Unpublished data.

Shaffer, D. M., \& McBeath, M. K. (2002). Baseball outfielders maintain a linear optical trajectory when tracking uncatchable fly balls. Journal of Experimental Psychology: Human Perception \& Performance, 28, 335-348.

Shaffer, D. M., McBeath, M. K., Roy, W. L., \& Krauchunas, S. M. (2003). A linear optical trajectory informs the fielder where to run to the side to catch fly balls. Journal of Experimental Psychology: Human Perception \& Performance, 29, 1244-1250.

Simmons, J. A., Fenton, M. B., \& O'Farrell, M. J. O. (1979). Echolocation and pursuit of prey by bats. Science, 203, 16-21.

Sugar, T. G., \& McBeath, M. K. (2001). Robotic modeling of mobile catching as a tool for understanding biological interceptive behavior. Brain \& Behavioral Sciences, 24, 1078-1080.

Suluh, A., Sugar, T. G., \& McBeath, M. K. (2001, May). Spatial navigational principles: Applications to mobile robotics. Paper presented at the IEEE International Conference on Robotics and Automation, Seoul, Korea.

Todd, J. T., Oomes, A. H. J., Koenderink, J. J., \& Kappers, A. M. L. (2001). On the affine structure of perceptual space. Psychological Science, 12, 191-196.

Vetter, P., Goodbody, S. J., \& Wolpert, D. M. (1999). Evidence for an eye-centered spherical representation of the visuomotor map. Journal of Neurophysiology, 81, 935-939

WatTs, R. G., \& BAHILL, A. T. (2000). Keep your eye on the ball: Curve balls, knuckleballs, and fallacies of baseball. New York: Freeman.

\section{NOTES}

1. The visual angle is measured with the point of origin in the stationary background scenery as $0,0^{\circ}$. Thus, the ball may still reach visual angles of $50^{\circ}-60^{\circ}$ while still being fixated by the fielder. As long as the subsequent position of the ball is coded as a result of previously sampled positions, this is consistent with the spherical projection sampling of optical ball position.

2. Balls were projected from a baseball-pitching machine with a launch angle of $63^{\circ}$. If not caught, the ball would land $13.9 \mathrm{~m}$ away from the machine. Fielders started randomly from one of four different positions relative to the eventual landing position of the ball (if it were not caught): $3 \mathrm{~m}$ left and $2 \mathrm{~m}$ forward; $3 \mathrm{~m}$ right and $4 \mathrm{~m}$ forward; $4 \mathrm{~m}$ left and $4 \mathrm{~m}$ backward; and $5 \mathrm{~m}$ right and $2 \mathrm{~m}$ backward.

(Manuscript received July 19, 2004; revision accepted for publication July 12, 2007.) 